\title{
The Role of Self-Action in 2-Year-Old Children: An Illustration of the Arithmetical Inversion Principle before Formal Schooling
}

\author{
Amélie Lubin, ${ }^{1,2,3}$ Sandrine Rossi, ${ }^{1,2,3}$ Nicolas Poirel, ${ }^{1,2,3,4}$ \\ Céline Lanoë, ${ }^{1,2,3}$ Arlette Pineau, ${ }^{1,2,3}$ and Olivier Houdé ${ }^{1,2,3,4}$ \\ ${ }^{1}$ CNRS, UMR 8240, LaPsyDÉ, 75000 Paris, France \\ ${ }^{2}$ Université Paris Descartes, Sorbonne Paris Cité, UMR 8240, LaPsyDÉ, 75000 Paris, France \\ ${ }^{3}$ Université de Caen Basse-Normandie, Normandie Université, UMR 8240, LaPsyDÉ, 14000 Caen, France \\ ${ }^{4}$ Institut Universitaire de France (IUF), 75000 Paris, France \\ Correspondence should be addressed to Amélie Lubin; amelie.lubin@parisdescartes.fr
}

Received 1 August 2014; Revised 30 January 2015; Accepted 1 February 2015

Academic Editor: Andrew N. Meltzoff

Copyright (C) 2015 Amélie Lubin et al. This is an open access article distributed under the Creative Commons Attribution License, which permits unrestricted use, distribution, and reproduction in any medium, provided the original work is properly cited.

\begin{abstract}
The importance of self-action and its considerable links with cognitive activity in childhood are known. For instance, in arithmetical cognition, 2-year-olds detected an impossible arithmetical outcome more accurately when they performed the operation themselves (actor mode) than when the experimenter presented it (onlooker mode). A key component in this domain concerns the understanding of the inversion principle between addition and subtraction. Complex operations can be solved without calculation by using an inversion-based shortcut (3-term problems of the form $a+b-b$ must equal $a$ ). Some studies have shown that, around the age of 4, children implicitly use the inversion principle. However, little is known before the age of 4 . Here, we examined the role of self-action in the development of this principle by preschool children. In the first experiment, 2-year-olds were confronted with inversion $(1+1-1=1$ or 2$)$ and standard $(3-1-1=1$ or 2$)$ arithmetical problems either in actor or onlooker mode. The results revealed that actor mode improved accuracy for the inversion problem, suggesting that self-action helps children use the inversion-based shortcut. These results were strengthened with another inversion problem $(1-1+1=1$ or 2$)$ in a second experiment. Our data provide new support for the importance of considering self-action in early mathematics education.
\end{abstract}

\section{Introduction}

A growing body of research is appearing in the field of embodied cognition [1-4]. Embodied cognition suggests that higher cognitive concepts may be based on bodily (i.e., action) experiences [5]. Self-actions modify how we think about the objects we encounter by interconnecting our representation of these objects with the sensory-motor experience associated with acting on objects [6]. The perspective that action and cognition are linked is, of course, not new in developmental psychology [4]. Piaget [7] first noted the influence of action on the development of intelligence. Seminal studies have shown the importance of motor activity and its considerable link with cognitive activity during childhood [2, 6, 8-14]. Embodied cognition is therefore highly relevant for education such as mathematics $[1,15]$. Indeed, the beneficial role of children's action in facilitating learning and thought has been highlighted in this domain [11, 13, 16-23]. In this study, we examined the role of embodied cognition, specifically the role of self-action (i.e., performing an action by oneself), in the development of the arithmetic principle in preschool children.

A key component in the development of arithmetic concerns the understanding of the inversion principle. Arithmetical inversion between addition and subtraction defines the relationship between these two operations: subtraction is the inverse of addition and vice versa. Indeed, adding a particular number $b$ to an array $a$ can be negated by subtracting the same number $b$ (i.e., $a+b-b$ must equal $a$ ). With this principle, a complex operation can be solved more efficiently by using a substitution method that is an inversionbased shortcut. The implicit use of this principle can be tested 
by determining whether children solve the inversion problem (e.g., calculation is not necessary to solve $78+25-25$ ) more accurately than a standard problem (e.g., calculation is required to solve $78+25-37$ ) of comparable size.

This topic has drawn considerable attention in recent years (see special issues $[24,25]$ ), with notable discussion on the age at which children have a full grasp of this principle [26]. This major controversy may be explained by the fact that the definition of the term "inversion" is not universally consensual and sometimes refers to different concepts [24]. According to Piaget's theory, the notion of arithmetical inversion is fundamental to mastering the nature of addition and subtraction. Children can understand this principle around the age of 6 or 7 years and can explain it later, around the age of 10 . Before the age of 6 , children can add and subtract without necessarily understanding the relationship between these operations $[27,28]$. Along these lines, Canobi and collaborators observed that 6- to 9-year-old children often fail to use the inversion principle even after formal instruction [29-31]. However, some studies have shown that before formal schooling, around the age of 4 , children implicitly use the inversion principle [32-36]. Some authors have argued that children seem to be sensitive to this principle even before the age of 4 $[37,38]$. In Starkey and Gelman's study [38], the experimenter placed some small objects in his hand and hid them. He added and subtracted other objects and asked the child how many he had in his hand. The children were presented with some inversion problems $(1+1-1,2+1-1$, and $3+1-1)$ and a standard problem $(2+2-1)$. The 3 -year-old children performed better on inversion problems than on the standard problem. Sherman and Bisanz [37] used a similar method. The experimenter presented an initial array of blocks $(a)$ to the children. Then, the experimenter covered the middle of the array and added a number of blocks $(b)$. For the inversion problems, $b$ blocks were removed. For standard problems, another number of blocks $(c)$ were removed. The children were asked to tell how many blocks remained in the array. The authors observed that children were better at the inversion problems. However, no significant correlation was obtained with children's counting performances. The authors argued that very young children could solve inversion problems before they became skillful counters. Although it was not the objective of Izard et al. [39] to study the use of the inversion principle, the transformation proposed in the identity condition of experiment 4 seemed to be an inversion problem. They used a nonverbal manual search task with 2-year-olds. An experimenter put 5 (or 6) puppets in an opaque box, took a puppet out of the box, and, then, after a short delay, returned the puppet to the box (i.e., $5-1+1$ ). Children were invited to retrieve the puppets from the box. In this identity condition, the children succeeded, suggesting the use of the inversion principle by 2 -year-olds. However, no standard problem was proposed here, as is usual in inversion experiments. Thus, it is difficult to draw accurate conclusions. Vilette [36] observed during a violation-of-expectation paradigm ([40], see also [41, 42]) that children grasp the inverse relationship between addition and subtraction only after the age of 4 . Two Babar dolls were presented in a little theatre, and the experimenter closed an opaque screen. One Babar doll was added and immediately removed, and the children were presented with either a possible, $2+1-1=2$, or an impossible, $2+1-1=3$, outcome. They were then asked whether each outcome was correct. Although the 4-year-olds succeeded, the younger children failed to detect the impossible outcome. Note that the author did not present a standard problem (in which calculation was necessary) to assess the use of the shortcut strategy, ruling out the possibility of determining whether 4-year-old children used the inversion-based shortcut or simply calculated.

Given the effects of the method on children's performance, it has been suggested that developmental psychologists must adapt their methods to the age of the children involved in the testing $[13,32,43,44]$. In the studies cited above [36-39], the experimenter performed the operations, and the children often responded verbally (except [39], which used a manual search). However, from Piaget's work, we know that, prior to language emergence, children often solve problems through action [45]. At this age, sensorymotor experiences are essential for gaining knowledge and developing cognitive abilities. Lubin and collaborators [13, 23] previously showed that allowing the child to be the actor during the experiment was effective in revealing early arithmetic abilities. They compared a violation-of-expectation method in which the experimenter presented the problem to the children (onlooker mode) with a new method based on action (actor mode) in which the children performed the operations by manipulating the material themselves. For addition problems $(1+1=1,2$, or 3$)$, the results revealed that 2-year-old children detected erroneous results more accurately in actor mode than in onlooker mode [13]. Moreover, the children had better performance in onlooker mode after they solved a problem in an actor mode, suggesting a pedagogical effect of action on arithmetic performance at this age [23]. This methodology, which allows children to perform the set-up of the displays, favors the demonstration of implicit knowledge of arithmetic principles.

According to the aforementioned beneficial effect of selfaction in arithmetical skills, we investigated the early roots of the inversion principle using the same methodology [13, 23]. We hypothesized that children might use the inversion principle to resolve 3-term problems more easily if they are the actors of the arithmetical operation. Indeed, embodying the inversion problem (to undo their self-action) should allow children to better integrate the cognitive shortcut based on inversion.

\section{Experiment 1}

The first study tested our assumption directly by presenting inversion $(1+1-1)$ and standard $(3-1-1) 3$-term arithmetic problems to 2-year-old children long before they attended formal schooling. We used a standard violation-ofexpectation paradigm with onlooker and actor modes (see [13]). If the children used calculation to solve the presented problems, then the inversion and standard problems should be equally difficult. Alternatively, if 2-year-old children are able to implicitly use the inversion principle, then accuracy should be better in the inversion problem than in the standard problem. Moreover, actor mode should allow young children 
to detect erroneous arithmetic results more easily during the inversion problem (adding $b$ and subtracting $b$ with selfaction) than simply observing an experimenter performing the same inversion problem (onlooker mode). In agreement with our previous results [13], only actor mode should allow children to spontaneously use the shortcut strategy that is cognitively less costly than calculation.

\subsection{Method}

2.1.1. Participants. Forty 2-year-old children recruited from childcare centers in Caen, France (mean age: 2 years and 8 months, range: 2 years and 5 months to 2 years and 11 months, 20 boys, middle-class homes, French native speakers), participated in this experiment. Written informed consent was obtained from parents.

2.1.2. Design and Procedure. The children were tested individually for approximately 10 minutes in a quiet room. In the "onlooker mode" group, the experimenter presented the arithmetic operations to the children, whereas, in the "actor mode" group, the children executed the same arithmetic operations themselves (see [13] for more details). Half of the participants were randomly assigned to each of these two presentation modes. Two arithmetic problems of similar magnitude were used: an inversion problem " $1+1-1=1$ or 2" (i.e., possible or impossible outcome) and a standard problem " $3-1-1=1$ or 2 ." In the inversion problem, one Babar doll was put in a box behind glass (by the experimenter or the child himself, according to the mode). Next, the screen was closed so that the children could no longer see the doll. One doll was then added to the first doll already in the box, and then one doll was removed $(1+1-1)$. In the standard problem, three dolls were added successively to the box. Next, the screen was closed, and two dolls were removed one at a time $(3-1-1)$. Each outcome (possible or impossible) was presented twice, and the order of the problem type was counterbalanced. In the two modes, the children were asked to determine which of the final outcomes (i.e., 1 or 2) was the correct result of the operation (Figure 1).

2.2. Results. All analyses were based on the participants' percentages of correct responses. Accuracy was analyzed with a 2 (mode: actor or onlooker) $\times 2$ (problem Type: inversion or standard) repeated-measures ANOVA. Post hoc comparisons were performed using Tukey's Honest Significant Difference tests.

The main effect of problem type showed that children were more accurate on the inversion problem (mean percent correct \pm standard error of the mean: $67.5 \pm 6.6 \%)$ than on the standard problem $(43.8 \pm 6.7 \%), F(1,38)=7.67, p<0.001$, and $\eta^{2} p=.17$. There was no main effect of mode of presentation $(61.3 \pm 6.8 \%$ and $50 \pm 6.9 \%$ for actor mode and onlooker mode, resp.), $F(1,38)=1.51, P=.23$, and $\eta^{2} p=0.04$. Finally, a twoway interaction of mode $\times$ problem Type, $F(1,38)=11.23$, $p=0.002$, and $\eta^{2} p=0.23$, revealed that, during actor mode, the 2-year-old children were better at the inversion problem $(87.5 \pm 6 \%)$ than at the standard problem $(35 \pm 9 \%)(p=$
0.0007 , Cohen's $d=1.55$ ). No difference was found between the two problem types during onlooker mode $(47.5 \pm 9 \%$ and $52.5 \pm 9 \%$ for inversion and standard problems, resp., $P=0.98$ ) (see Figure 2). Note that the children were better at the inversion problem in actor mode $(87.5 \pm 6 \%)$ than in onlooker mode ( $47.5 \pm 9 \%, p<0.05$, Cohen's $d=1.08)$.

2.3. Discussion. The present study is the first to document the role of self-action in 2-year-old children on the arithmetical inversion principle before formal schooling. The results revealed that, in comparison to the traditional "onlooker" method (i.e., when arithmetic problems were presented to the children by the experimenter), 2-year-old children used inversion-based shortcuts when they performed the arithmetic 3-term problems themselves in actor mode. In particular, although their performances were not different from chance on the standard problem $\left(\chi^{2}, p>0.05\right)$, the children succeeded on the inversion problem. Taken together, these results suggest that the actor method (1) seems to be an effective procedure for testing young children and (2) reveals the true arithmetic inversion abilities of 2-year-olds. By reversing their own action, young children may have implicitly grasped the inverse strategy and detected the impossible event more easily during the inversion problem. Conversely, the only successful strategy for the standard problem was to calculate the result, and the children's performance showed that this operation was certainly beyond their competencies (due to the absence of formal learning).

Interestingly, the children's performance on both problems did not differ from the level of chance $\left(\chi^{2}, p>\right.$ 0.05 ) during onlooker mode, indicating that these 3-term problems were probably too difficult for 2-year-old children. This result reinforces the assumption that observing someone performing the problems does not allow children to see the inverse strategy and does not promote their success on the standard problem. Taken together, our results suggest that onlooker mode may not always be adaptable to studies of toddlers, explaining why, for instance, no inverse principle was evidenced before the age of 4 in a previous study [36].

Because of the theoretical importance of these findings, we conducted an additional study with another problem in a new sample of 2-year-old children to assess the robustness of our results. In the first study, the children performed only 2 trials of each problem because it is difficult to maintain young children's attention over a long period. Moreover, because working memory constrains arithmetic performance in preschoolers [34], variation in problems is limited at this age. We decided to propose a new inversion problem $(1-1+$ $1=1$ or 2 ; the magnitude is the same, but subtraction appears before addition) and the same standard problem $(3-1-1=1$ or 2) with exactly the same design as in Experiment 1.

\section{Experiment 2}

\subsection{Method}

3.1.1. Participants. Forty more 2-year-old children were recruited from childcare centers in Caen, France (mean age: 2 years and 6 months, range: 2 years and 1 month to 2 years 


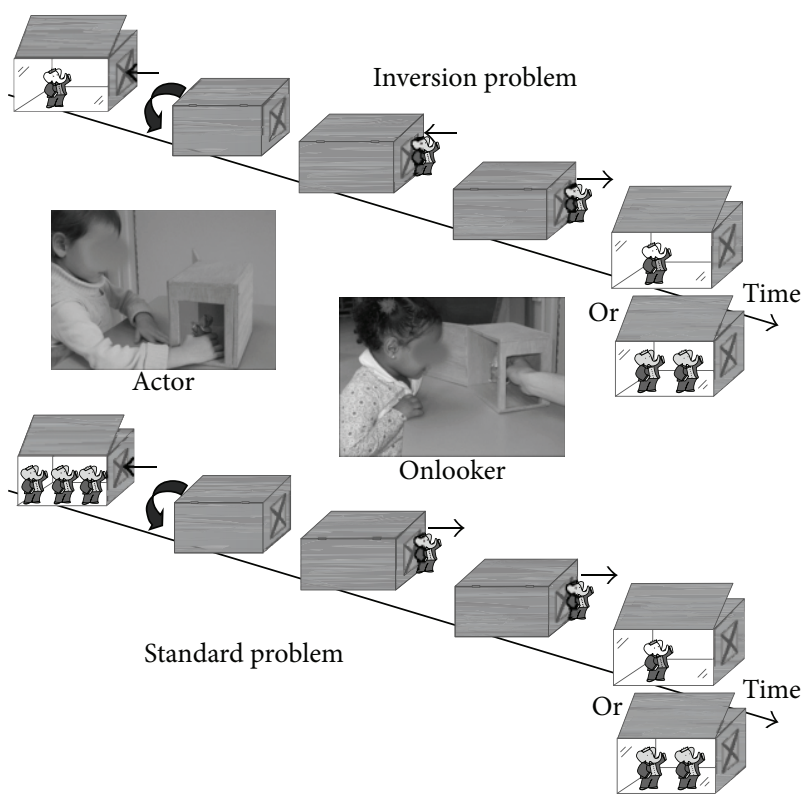

Figure 1: Presentation of the inversion problem $(1+1-1=1$ or 2 , top) and the standard problem ( $3-1-1=1$ or 2 , bottom) in either actor or onlooker modes.

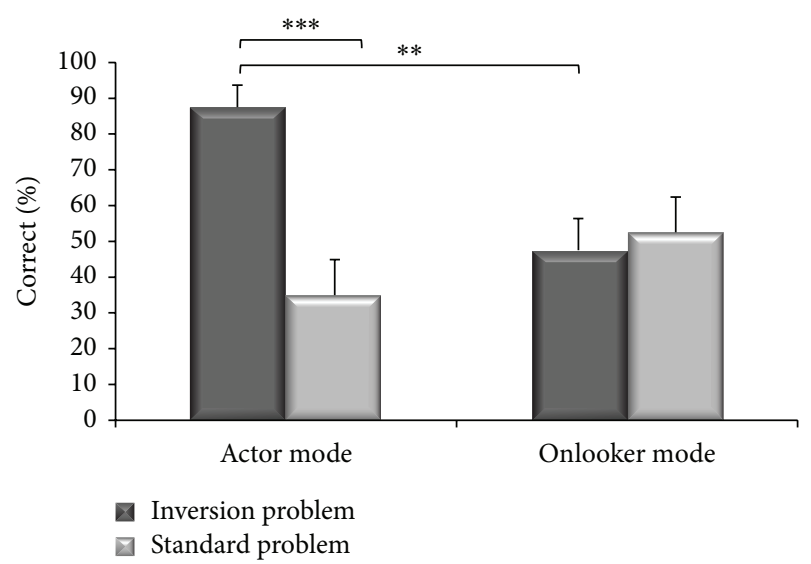

FIGURE 2: Percentage of correct responses as a function of problem type and mode. ${ }^{* * *} p<0.001 ;^{* *} p<0.01$.

and 11 months, 20 boys, middle-class homes, French native speakers), to participate in this experiment. Written informed consent was obtained from parents.

3.1.2. Design and Procedure. The apparatus was the same as in Experiment 1 . Half of the children were randomly assigned to one of two presentation modes (onlooker mode or actor mode), and we introduced a new inversion problem. One Babar doll was put in a box behind glass (by the experimenter or the child himself, according to the mode). Next, the screen was closed so that the children could no longer see the doll. The doll was removed, and, then, one doll was added $(1-1+$
1). The standard problem was the same as in Experiment 1 $(3-1-1)$.

3.2. Results. All analyses were based on the participants' percentages of correct responses. Accuracy was analyzed with a 2 (mode: actor or onlooker) $\times 2$ (problem type: inversion or standard) repeated-measures ANOVA.

The main effect of problem type showed that children were more accurate on the inversion problem (mean percent correct \pm standard error of the mean: $53.8 \pm 6.3 \%)$ than on the standard problem $(33.8 \pm 5.8 \%), F(1,38)=10.12, p<$ 0.005 , and $\eta^{2} p=0.21$. There was a main effect of mode of presentation $(55 \pm 6.7 \%$ and $32.5 \pm 5.2 \%$ for actor mode and onlooker mode, resp.), $F(1,38)=5.75, p=0.02$, and $\eta^{2} p=.13$. Finally, there was no two-way interaction mode $\times$ problem type, $F(1,38)=2.55, P=.12$, and $\eta^{2} p=0.06$. In the absence of interaction, we performed planned comparison. As in Experiment 1, during actor mode, the 2-year-old children were better at the inversion problem $(70 \pm 8.4 \%)$ than at the standard problem $(40 \pm 9.3 \%)(p=0.002$, Cohen's $d=0.8)$. No difference was found between the two problem types during onlooker mode $(37.5 \pm 8 \%$ and $27.5 \pm 6.8 \%$ for inversion and standard problems, resp., $P=0.27)$. Note that the children were better at the inversion problem in actor mode $(70 \pm 8.4 \%)$ than in onlooker mode $(37.5 \pm 8 \%, p<0.01$, Cohen's $d=0.9)$.

3.3. Discussion. The findings of Experiment 2 reinforced those of Experiment 1 with another inversion problem on a new sample of participants. We pooled all the data to reinforce the robustness of our findings. This new analysis performed with 80 2-year-old children accorded with the original findings. We obtained a main effect of problem type $F(1,78)=16.65, p<0.001$, and $\eta^{2} p=.17$, a main effect of mode of presentation $F(1,78)=6.47, p=0.01$, and $\eta^{2} p=$ 0.07 , and, most crucially, an interaction between these two factors $F(1,78)=13.09, p<0.001$, and $\eta^{2} p=0.14$. Children were better at the inversion problem $(79 \pm 5.3 \%)$ than at the standard problem $(37.5 \pm 6.4 \%)$ in actor mode $(p<0.001$, Cohen's $d=1.1)$. No difference was found between these two problem types in onlooker mode ( $42.5 \pm 6.3 \%$ and $40 \pm 6.3 \%$ for inversion and standard problems, resp., $P=0.98$ ).

\section{General Discussion}

Our results show for the first time that sensitivity to the inversion principle appears as early as 2 years of age. Although 2 -year-old children were previously characterized as "bad counters" [37], they seemed to be able to use the inversion principle by enacting it. As previously suggested for simple arithmetic operations $[13,23]$, the present study suggests that a paradigm based on action seems better adapted for evaluating the numerical abilities of preschoolers. This finding underlines the importance of adapting the method to the age of children $[13,32,43,44]$ because low performance does not necessarily reveal a lack of competency and, in fact, depends on the method of testing. 
Our results are in agreement with embodied mathematical cognition $[16,18,21]$. Self-action reveals new strategies and brings out implicit knowledge $[17,46]$. In this line, embodied intervention programs have shown beneficial effects in the mathematical domain [11, 22]. However, one could ask what exactly the role of action is. Are children discovering the inversion principle during the experiment, or is it the method that reveals preexisting knowledge? We believe that embodying the inversion problem (undoing their self-action) allows children to better integrate the cognitive shortcut based on inversion and could reactivate implicit knowledge discovered by the active exploration of their environment. For instance, children can recognize in everyday life experience that putting one candy in a box and removing another candy from the same box leaves the initial amount of candies unchanged. However, children were unable to detect the shortcut when they only observed it. Our paradigm based on self-action activates (or reactivates) the inversion strategy and leads to success with the inversion problem.

Studying early arithmetic development before the onset of formal schooling provides crucial insights into young children's emerging academic learning and could be useful for adapting pedagogical methods. Before they enter school, children use mathematical notions in everyday life and develop informal mathematical knowledge. However, with the emergence of numerical symbolization, children must reorganize their early knowledge through this new format. Thus, there is often an apparent gap between children's informal, intuitive knowledge and the formal opportunities at the start of their schooling [47]. Therefore, studying ways to support and reinforce the development of early numerical abilities could be critical for bridging intuitive mathematical knowledge and formal schooling and for better understanding (and optimizing) the transition between them. Educators and teachers should find ways to help children link what they know implicitly and what they must learn at school [48].

In the particular case of the inversion principle, our findings suggest that an implicit understanding of the inversion principle precedes explicit formal learning. However, a gap has been observed between the presence of the inversion strategy in preschool children (as emphasized here) and its use in school-aged children [49], suggesting that the link between implicit and explicit inversion is not explicitly made at school. Nevertheless, teaching the inverse relationship between addition and subtraction can improve the understanding and the use of inversion between the ages of 4 and 9 years [50-53]. These mathematical ideas could be useful for preschool teachers to move children towards more formal knowledge by encouraging mathematical exploration in many contexts and by inciting young children to perform inversions in different classroom contexts. Self-action, as an index of transition, may help children to solidify the connection between the numerical symbolic system and their nonsymbolic knowledge.

\section{Conclusion}

The present results revealed that actor mode improved the accuracy of 2-year-olds for inversion problems, suggesting, for the first time, that self-action helps children use the inversion-based shortcut during this developmental window. The present work provides new support for the view that it may be worthwhile to consider the self-action advantage in early mathematics education.

\section{Conflict of Interests}

The authors declare that there is no conflict of interests regarding the publication of this paper.

\section{Acknowledgments}

The authors thank the children and parents who took part in this experiment and the staff of the childcare centers for their warm welcome. They thank the anonymous reviewers for their very useful comments and suggestions.

\section{References}

[1] M. Kiefer and N. M. Trumpp, "Embodiment theory and education: the foundations of cognition in perception and action," Trends in Neuroscience and Education, vol. 1, no. 1, pp. 15-20, 2012.

[2] C. Kontra, S. Goldin-Meadow, and S. L. Beilock, "Embodied learning across the life span," Topics in Cognitive Science, vol. 4, no. 4, pp. 731-739, 2012.

[3] D. H. Rakison and A. L. Woodward, "New perspectives on the effects of action on perceptual and cognitive development," Developmental Psychology, vol. 44, no. 5, pp. 1209-1213, 2008.

[4] M. Wellsby and P. M. Pexman, "Developing embodied cognition: insights from children's concepts and language processing," Frontiers in Psychology, vol. 5, article 506, 2014.

[5] M. Wilson, "Six views of embodied cognition," Psychonomic Bulletin \& Review, vol. 9, no. 4, pp. 625-636, 2002.

[6] S. Goldin-Meadow and S. L. Beilock, "Action's influence on thought: the case of gesture," Perspectives on Psychological Science, vol. 5, no. 6, pp. 664-674, 2010.

[7] J. Piaget, The Origins of Intelligence in Children, Norton \& Co., New York, NY, USA, 1952.

[8] B. G. Bender and J. R. Levin, "Motor activity, anticipated motor activity, and young children's associative learning," Child development, vol. 47, no. 2, pp. 560-562, 1976.

[9] M. H. Bornstein, C.-S. Hahn, and J. T. D. Suwalsky, "Physically developed and exploratory young infants contribute to their own long-term academic achievement," Psychological Science, vol. 24, no. 10, pp. 1906-1917, 2013.

[10] D. Buttelmann, M. Carpenter, and M. Tomasello, "Eighteenmonth-old infants show false belief understanding in an active helping paradigm," Cognition, vol. 112, no. 2, pp. 337-342, 2009.

[11] U. Fischer, K. Moeller, M. Bientzle, U. Cress, and H.-C. Nuerk, "Sensori-motor spatial training of number magnitude representation," Psychonomic Bulletin \& Review, vol. 18, no. 1, pp. 177-183, 2011.

[12] A. Frick, M. M. Daum, M. Wilson, and F. Wilkening, "Effects of action on children's and adults' mental imagery," Journal of Experimental Child Psychology, vol. 104, no. 1, pp. 34-51, 2009.

[13] A. Lubin, N. Poirel, S. Rossi, A. Pineau, and O. Houdé, "Math in actions: actor mode reveals the true arithmetic abilities of 
French-speaking 2-year-olds in a magic task," Journal of Experimental Child Psychology, vol. 103, no. 3, pp. 376-385, 2009.

[14] J. Piaget and B. Inhelder, The Child's Conception of Space, Norton, New York, NY, USA, 1967.

[15] T. Ionescu and D. Vasc, "Embodied cognition: challenges for psychology and education," Procedia-Social and Behavioral Sciences, vol. 128, pp. 275-280, 2014.

[16] J. Bahnmueller, T. Dresler, A.-C. Ehlis, U. Cress, and H.C. Nuerk, "NIRS in motion-unraveling the neurocognitive underpinnings of embodied numerical cognition," Frontiers in Psychology, vol. 5, article 743, 2014.

[17] S. C. Broaders, S. W. Cook, Z. Mitchell, and S. Goldin-Meadow, "Making children gesture brings out implicit knowledge and leads to learning," Journal of Experimental Psychology: General, vol. 136, no. 4, pp. 539-550, 2007.

[18] F. Domahs, K. Moeller, S. Huber, K. Willmes, and H.-C. Nuerk, "Embodied numerosity: implicit hand-based representations influence symbolic number processing across cultures," Cognition, vol. 116, no. 2, pp. 251-266, 2010.

[19] S. Goldin-Meadow, S. W. Cook, and Z. A. Mitchell, "Gesturing gives children new ideas about math," Psychological Science, vol. 20, no. 3, pp. 267-272, 2009.

[20] S. Goldin-Meadow, H. Nusbaum, S. D. Kelly, and S. Wagner, "Explaining math: gesturing lightens the load," Psychological Science, vol. 12, no. 6, pp. 516-522, 2001.

[21] R. Hall and R. Nemirovsky, "Introduction to the special issue: modalities of body engagement in mathematical activity and learning," Journal of the Learning Sciences, vol. 21, no. 2, pp. 207$215,2012$.

[22] T. Link, K. Moeller, S. Huber, U. Fischer, and H.-C. Nuerk, "Walk the number line-an embodied training of numerical concepts," Trends in Neuroscience and Education, vol. 2, no. 2, pp. 74-84, 2013.

[23] A. Lubin, N. Poirel, S. Rossi, C. Lanoë, A. Pineau, and O. Houdé, "Pedagogical effect of action on arithmetic performances in Wynn-like tasks solved by 2-year-olds," Experimental Psychology, vol. 57, no. 6, pp. 405-411, 2010.

[24] A. J. Baroody, J. Torbeyns, and L. Verschaffel, "Young children's understanding and application of subtraction-related principles," Mathematical Thinking and Learning, vol. 11, no. 1-2, pp. 2-9, 2009.

[25] L. Verschaffel, P. Bryant, and J. Torbeyns, "Introduction," Educational Studies in Mathematics, vol. 79, no. 3, pp. 327-334, 2012.

[26] C. K. Gilmore and M. Papadatou-Pastou, "Patterns of individual differences in conceptual understanding and arithmetical skill: a meta-analysis," Mathematical Thinking and Learning, vol. 11, no. 1-2, pp. 25-40, 2009.

[27] J. Piaget, The Child's Conception of Number, Routledge \& Kegan Paul, London, UK, 1952.

[28] J. Piaget and A. Moreau, "The inversion of arithmetic operations," in Studies in Reflecting Abstraction, J. Piaget and R. L. Campell, Eds., pp. 69-86, Psychology Press, Hove, UK, 2001.

[29] K. H. Canobi, "Individual differences in children's addition and subtraction knowledge," Cognitive Development, vol. 19, no. 1, pp. 81-93, 2004.

[30] K. H. Canobi, “Children's profiles of addition and subtraction understanding," Journal of Experimental Child Psychology, vol. 92, no. 3, pp. 220-246, 2005.

[31] K. H. Canobi, R. A. Reeve, and P. E. Pattison, "The role of conceptual understanding in children's addition problem solving," Developmental Psychology, vol. 34, no. 5, pp. 882-891, 1998.
[32] P. Bryant, C. Christie, and A. Rendu, “Children's understanding of the relation between addition and subtraction: inversion, identity, and decomposition," Journal of Experimental Child Psychology, vol. 74, no. 3, pp. 194-212, 1999.

[33] C. K. Gilmore and E. S. Spelke, "Children's understanding of the relationship between addition and subtraction," Cognition, vol. 107, no. 3, pp. 932-945, 2008.

[34] J. S. Klein and J. Bisanz, "Preschoolers doing arithmetic: the concepts are willing but the working memory is weak," Canadian Journal of Experimental Psychology, vol. 54, no. 2, pp. 105-115, 2000.

[35] C. Rasmussen, E. Ho, and J. Bisanz, "Use of the mathematical principle of inversion in young children," Journal of Experimental Child Psychology, vol. 85, no. 2, pp. 89-102, 2003.

[36] B. Vilette, "Do young children grasp the inverse relationship between addition and subtraction? Evidence against early arithmetic," Cognitive Development, vol. 17, no. 3-4, pp. 1365-1383, 2002.

[37] J. Sherman and J. Bisanz, "Evidence for use of mathematical inversion by three-year-old children," Journal of Cognition and Development, vol. 8, no. 3, pp. 333-344, 2007.

[38] P. Starkey and R. Gelman, "The development of addition and subtraction abilities prior to formal schooling in arithmetic," in Addition and Subtraction: A Cognitive Perspective, T. P. Carpenter, J. M. Moser, and T. A. Romberg, Eds., pp. 99-116, LEA, Hillsdale, NJ, USA, 1982.

[39] V. Izard, A. Streri, and E. S. Spelke, "Toward exact number: young children use one-to-one correspondence to measure set identity but not numerical equality," Cognitive Psychology, vol. 72, pp. 27-53, 2014.

[40] O. Houdé, "Numerical development: from the infant to the child. Wynn's (1992) paradigm in 2- and 3-year olds," Cognitive Development, vol. 12, no. 3, pp. 373-391, 1997.

[41] A. Lubin, A. Pineau, C. Hodent, and O. Houdé, "Languagespecific effects on number computation in toddlers: a European cross-linguistic cartography," Cognitive Development, vol. 21, no. 1, pp. 11-16, 2006.

[42] K. Wynn, "Addition and subtraction by human infants," Nature, vol. 358, no. 6389, pp. 749-750, 1992.

[43] C. K. Gilmore and P. Bryant, "Individual differences in children's understanding of inversion and arithmetical skill," British Journal of Educational Psychology, vol. 76, no. 2, pp. 309-331, 2006.

[44] J. Langer, P. Gillette, and R. I. Arriaga, “Toddlers' cognition of adding and subtracting objects in action and in perception," Cognitive Development, vol. 18, no. 2, pp. 233-246, 2003.

[45] J. Piaget, "Piaget's theory," in Handbook of Child Psychology, P. H. Mussen, Ed., pp. 103-128, Wiley, New York, NY, USA, 1984.

[46] R. Boncoddo, J. A. Dixon, and E. Kelley, "The emergence of a novel representation from action: evidence from preschoolers," Developmental Science, vol. 13, no. 2, pp. 370-377, 2010.

[47] D. H. Clements and J. Samara, "Early childhood mathematics learning," in Second Handbook on Mathematics Teaching and Learning, F. K. Lester Jr., Ed., pp. 461-555, Information Age, Charlotte, NC, USA, 2007.

[48] M. Schneider and E. Stern, "The inverse relation of addition and subtraction: a knowledge integration perspective," Mathematical Thinking and Learning, vol. 11, no. 1-2, pp. 92-101, 2009.

[49] J. Bisanz, R. P. D. Watchorn, C. Piatt, and J. Sherman, "On 'understanding' children's developing use of inversion," Mathematical Thinking and Learning, vol. 11, no. 1-2, pp. 10-24, 2009. 
[50] M. L. Lai, A. J. Baroody, and A. R. Johnson, "Fostering Taiwanese preschoolers' understanding of the addition-subtraction inverse principle," Cognitive Development, vol. 23, no. 1, pp. 216-235, 2008.

[51] T. Nunes, P. Bryant, D. Evans, D. Bell, and R. Barros, "Teaching children how to include the inversion principle in their reasoning about quantitative relations," Educational Studies in Mathematics, vol. 79, no. 3, pp. 371-388, 2012.

[52] T. Nunes, P. Bryant, D. Hallett, D. Bell, and D. Evans, “Teaching children about the inverse relation between addition and subtraction," Mathematical Thinking and Learning, vol. 11, no. 1-2, pp. 61-78, 2009.

[53] K. M. Robinson and A. K. Dubé, "Children's use of arithmetic shortcuts: the role of attitudes in strategy choice," Child Development Research, vol. 2012, Article ID 459385, 10 pages, 2012. 

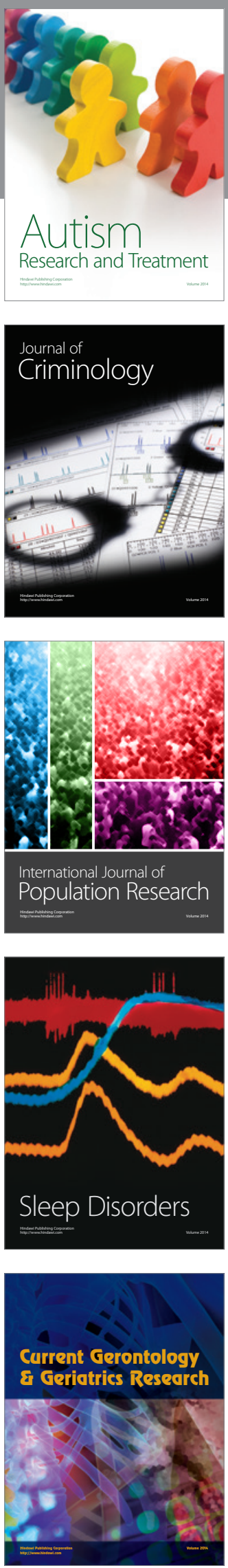
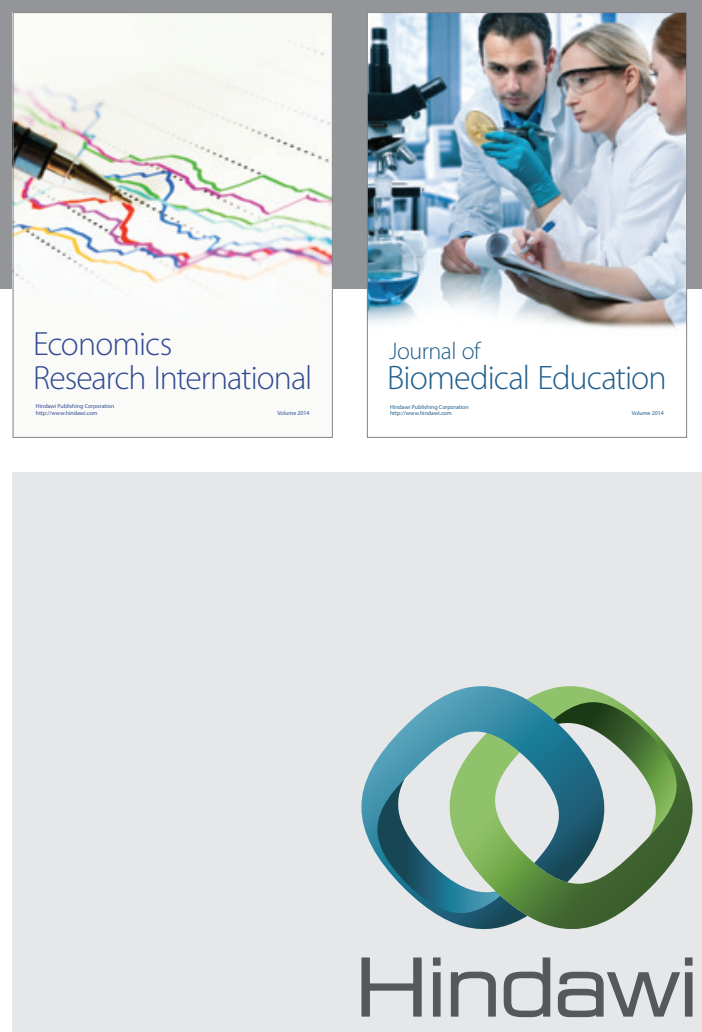

Submit your manuscripts at

http://www.hindawi.com
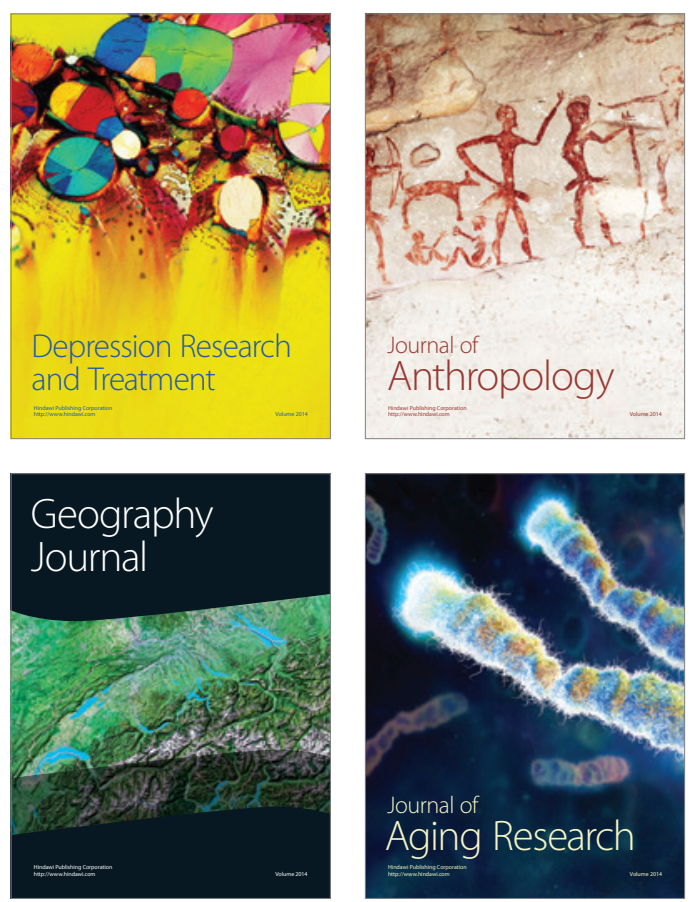
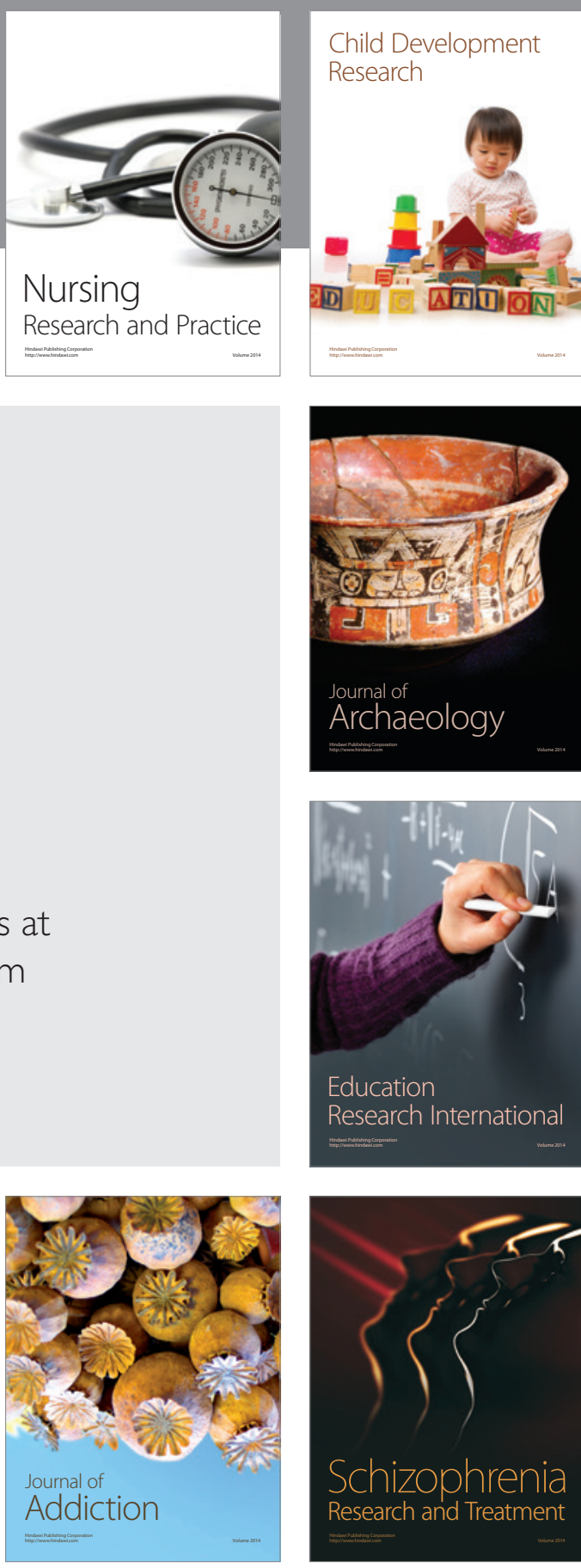

(D)
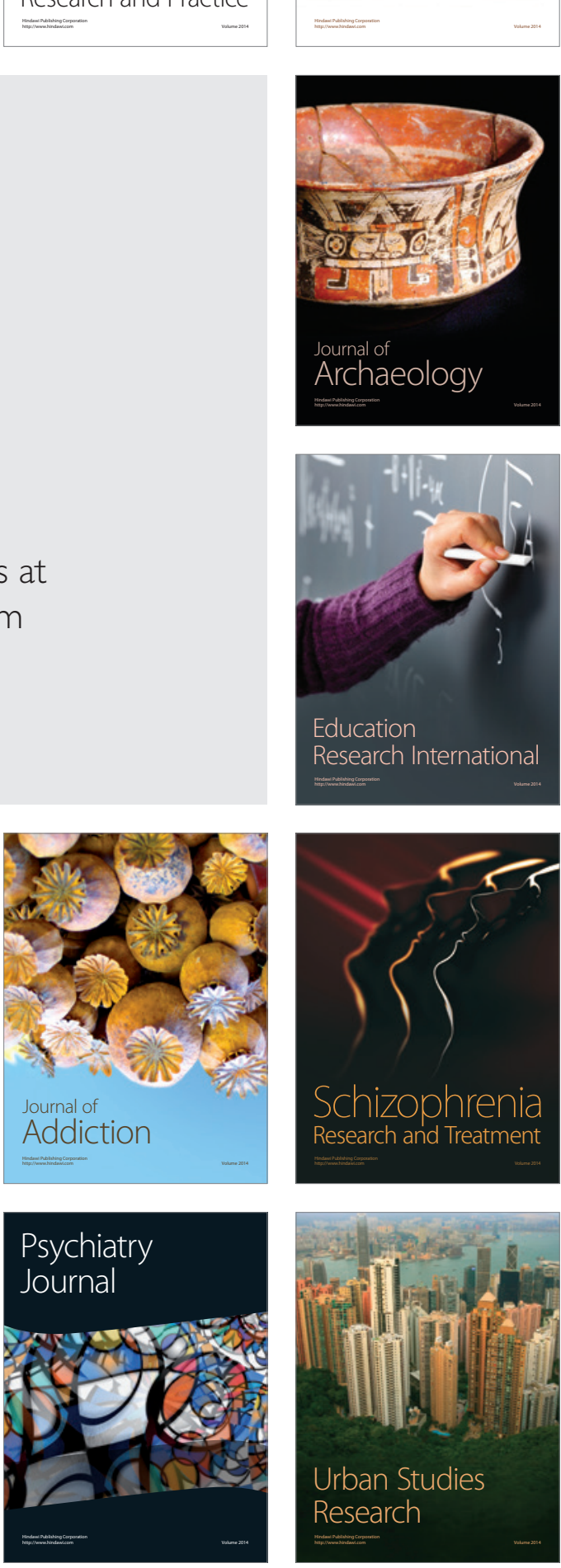